\title{
Retrospective Assessment of Drug Prescription and Usage Pattern among Pregnant Women under Ante Natal Care in Swaziland
}

\author{
Adefolarin A Amu ${ }^{1}$, Nolwazi M Ndzimande ${ }^{1}$, Nolwazi N Tfwala $^{1}$ and \\ Julius 0. Soyinka1, 2* \\ 1Department of Pharmacy, Swaziland Christian University, Swaziland \\ 2Department of Pharmaceutical Chemistry, Faculty of Pharmacy, Obafemi Awolowo \\ University, Ile-Ife, Nigeria
}

\section{Research Article \\ Volume 3 Issue 2}

Received Date: June 15, 2018

Published Date: June 26, 2018

*Corresponding author: Julius O. Soyinka, Department of Pharmaceutical Chemistry, Faculty of Pharmacy, Obafemi Awolowo University, Ile-Ife, Nigeria, Tel: +2348035822785; Email: juliussoyinka@gmail.com

\section{Abstract}

Background: Inappropriate drug use during pregnancy brings possible teratogenicity to the foetus, however chronic illnesses, like diabetes, and pregnancy induced sicknesses, like nausea and vomiting, with consideration of the risk benefit ratio has necessitated the increased use of medication during pregnancy, leading to adverse reproductive outcomes. There is increased statistics of maternal mortality, neonatal deaths, infant mortality, induced labour, miscarriages, premature labour/stillbirth, and other pregnancy complications in Swaziland, hence, the study.

Objective: To assess drug prescribing and usage pattern among pregnant women who attended antenatal care at a selected General Hospital between January and December 2016.

Method: A quantitative retrospective non-experimental survey design was used. The study was carried out at a selected General Hospital in the Manzini region of Swaziland. The population were pregnant women that attended antenatal care. Data was collected by reviewing pregnant patients' files from January 2016 to December 2016. Simple random sampling was used and the data was analysed using SPSS system version 20.0, and the US FDA risk category system was a reference standard.

Results: Out of the sample of 314 pregnant patient files reviewed it was observed that most patients were aged 21-30 202 (64.3\%), a majority of the patients were at their third trimester 170 (54.1\%), common diagnosis/complications being lower abdominal pain 105 (33.4\%) and urinary tract infection 101 (32.2\%). Nutritional supplements 682 (64.9\%), antimicrobials $188(17.9 \%)$ and analgesics 127 (12.1\%) were the most prescribed and used drugs. The most prescribed and used medication fell under category A 686 (64.9\%) followed by category B 287 (27.3\%), category C 64 (6.1\%), category D 17 (1.6\%), and category X $1(0.1 \%)$. 


\section{Advances in Pharmacology and Clinical Trials}

Conclusion: Since the most prescribed and used drugs were from category A and B, the safest categories according to by the US FDA risk category system, the overall drug prescribing and use pattern for the period in view was rational.

Keywords: Drug usage; Pregnancy; Prescribing pattern; Retrospective analysis

\section{Introduction}

Pregnancy is a complex state during which changes in physiology of the maternal organism have evolved to enhance development and growth of the placenta and foetus [1]. Beyond changes in cardiovascular, pulmonary, gastrointestinal, renal, and hepatic function during pregnancy, there are also changes in the expression and activity of transport proteins and enzyme systems, such as cytochrome P450 (CYP) enzymes leading to pharmacokinetic alterations which may change the metabolic profile of a drug [2]. These changes also alter maternal disposition of drugs and the effect of a drug on the mother, placenta, and foetus. Thus posing challenges to the clinicians when managing disease states during pregnancy and in the selection of medications best suited to treat the patients [1].

Drug use during pregnancy may pose a teratogenic risk. However, avoiding all drugs during pregnancy may be dangerous to the mother's health and indirectly to that of the foetus. Early accidental/intentional medication exposure during pregnancy is of concern because use of certain medicines in pregnancy can lead to congenital abnormalities in the foetus and other harmful effects [3]. Complications of preterm birth are the single largest direct cause of neonatal deaths, responsible for 35\% of the world's 3.1 million deaths a year, and the second most common cause of under-5 deaths after pneumonia [1].

According to the March of Dimes, approximately 150,000 babies are born with birth defects annually in the United States [4]. A study done in Ethiopia indicated that the concern about the safety of medicines being prescribed to pregnant women became increasingly evident since the thalidomide tragedy in the 1960s [3]. Thalidomide was used in the treatment of different disorders such as anxiety, insomnia, gastritis and tension, it was furthermore promoted as a safe antiemetic medication during pregnancy. The medicine was withdrawn from the market a few years after its introduction due to severe teratogenic effects including limb defects, ear malformations or hearing loss, and ocular abnormalities [3].
Globally, maternal mortality is unacceptably high. A study done in Kenya showed that an estimated maternal mortality ratio of 530 deaths per 100,000 live births, slightly lower than the average of 640 for sub-Saharan Africa. Each year, an estimated 7,900 Kenyan women die during or after pregnancy [5]. The International Safe Motherhood Conference revealed that 1400 women die every day in the process of carrying or delivering their children [6]. In Africa about half a million women die from maternal causes every year leaving behind at least a million motherless children [7].

According to Solarsh and Hofman, [8], it has been noted that like in other countries, neonatal mortality now accounts for $45 \%$ of infant deaths in South Africa. In Swaziland, the Ministry of Health revealed that stillbirth accounts for $60 \%$ of perinatal deaths [9]. A study done in Swaziland states that still birth rate for the four year period 1989 to 1992 was 20 per 1000 birth at one regional hospital [6]. Maternal mortality ratio (MMR) continues to be high in Swaziland compared to other countries in Sub-Saharan Africa [9]. In 2007, the country recorded 587 deaths per 100000 live births, showing an increase from 370 deaths in 1995. Statistics of 2013 on annual number of neonatal deaths was 1,094 of the population which indicates a high rate of neonatal deaths [10].

Increased statistics of maternal and infant mortality, neonatal deaths, induced labour, miscarriages, premature labour/stillbirth, and other pregnancy complications raised concern, thus was the need for assessment of drug prescribing and usage pattern in pregnant women which will give us an indication if such complications are due to the prescribing and drug usage pattern in antenatal care.

Health providers should be aware of appropriate medical therapy for a variety of conditions and potential impact on pregnant women and foetus. To safe guide drug use during pregnancy, the United States (US) Food and Drug Administration (FDA) classified drugs into five categories : $\mathrm{A}, \mathrm{B}, \mathrm{C}, \mathrm{D}$, and $\mathrm{X}$ with category $\mathrm{D}$ and $\mathrm{X}$ indicating evidence of risk in pregnancy [11]. 


\section{Advances in Pharmacology and Clinical Trials}

Maternal mortality and perinatal mortality are top causes of death among women of reproductive age in developing countries. Approximately, 350,000 women die each year due to pregnancy-related causes [9]. An early assessment of the situation will enhance prescribers' knowledge of the risk factors to consider when prescribing medication to pregnant women and also help in the proper usage of drugs.

Findings from this study will help to reduce, and prevent death and complications during gestation, such as preterm birth. It will further equip clinicians on the rational use of medications in pregnancy. It will also help provide individual pregnant woman with important medication information. Quality care during pregnancy will ensure all women have a positive pregnancy experience.

The purpose of the study is to assess the drug usage pattern in pregnant women attending antenatal care at a selected hospital in the Manzini Region of Swaziland, from January 2016 to December 2016 and to determine the risk level of pregnancy medication according to the US FDA risk category system.

\section{Methodology}

\section{Study Approach and Design}

The research was done using a quantitative approach. This involved previewing of pregnant patient's files. In this approach, a retrospective, descriptive, nonexperimental survey design was used.

\section{Study Setting}

The study was carried out at Raleigh Fitkin Memorial Hospital (RFM) in Manzini region of Swaziland. RFM is a regional referral and teaching hospital situated in Manzini, it is the hub of Swaziland that occupies 7,000 square meters in clinical buildings. Manzini is the most populous region in Swaziland. The hospital provides services to around 500 patients in the outpatient department daily. RFM has the highest number of hospital deliveries in the country with approximately one baby born every hour.

\section{Study population}

Population is a well-defined group of people or other entities that have specified properties [12]. In this study the population were pregnant women attending antenatal care in RFM.

\section{Sampling and Sample Method}

Sampling is the process of selecting representative units of the population for study in a research investigation [12]. Statistical techniques, formulas and analyses were used to find the correct sample size calculation accurately. After determining the sample size, pregnant women files were selected using the simple random sampling technique.

The sample size was determined based on the prevalence of drug use during pregnancy. According to a study done by Kebede, et al. [13], the prevalence of prescription drug use during pregnancy in Ethiopia was $71.3 \%$. Through the use of a single proportion formula taking the prevalence of the drug use during pregnancy as $73.3 \%$.

The total sample was 314. Sample size is concerned with how much data is required to make a correct decision on particular research. If there is more data, then the study will be more accurate and there will be less error of the parameter estimate.

Variables

1. Dependent variables

2. Diagnosis/complications, prescribed drugs, and risk category of the drug.

Independent variable

3. Number of ANC visit of patient, Age of patient, Trimesters.

4. Inclusion criteria and Exclusion criteria

5. Pregnant women who attended antenatal care at RFM Hospital. There were no exclusion criteria because every woman that attended antenatal care at RFM Hospital was pregnant.

\section{Ethical Clearance and Consideration}

The Ethical clearance was obtained from the Swaziland Research and Ethics Committee under the Ministry of Health, through recommendation from the Department of Pharmacy, permission was sought in writing and submitted to the Senior Medical Officer (SMO) of RFM with the ethical clearance attached. Patient confidentiality was kept by not documenting the names of the patients and retrieving the information or patient files in a private room away from the public or people not part of the research team. The hospital management committee and staff were fully informed about the purpose, methods, and intended use of the research. 


\section{Advances in Pharmacology and Clinical Trials}

\section{Data Collection and Analysis}

Data was collected by reviewing pregnant patients' prescription files from January 2016 to December 2016 and comparing the prescriptions with the US FDA risk category system as the standard. All the data from the patients' prescription files were coded using a code book and was entered into statistical package for social sciences (SPSS) version 20 statistical software for analysis. Efforts were made by the researchers to ensure efficient handling of data by using codes rather than participants' names, first by checking data by frequencies in order to spot missing or incorrect values. The data was also analyzed according to the US FDA risk category system.

\section{Limitation of the Study}

The study provided neither information on drugs dispensed over-the-counter (self-medication), it was only limited to the prescribed drugs, which were documented on the ANC follow up files. It was limited to an urban area and only one out of four regions of Swaziland, and it did not assess the practice of drug use in rural community, so generalization of the result was not possible.

\section{Results}

A total of 314 pregnant patients' files with complete and pertinent information were randomly selected and reviewed.

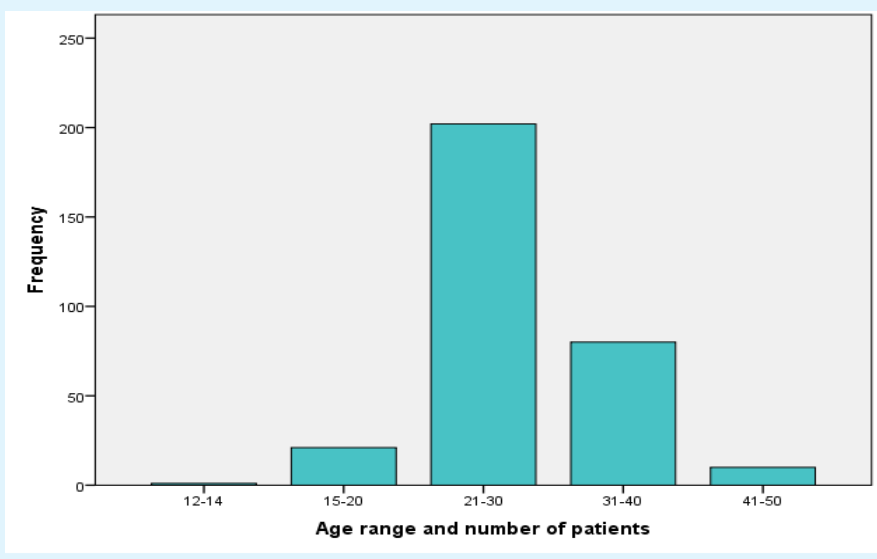

Figure 1: A graph showing the age range and number of patients against frequency.

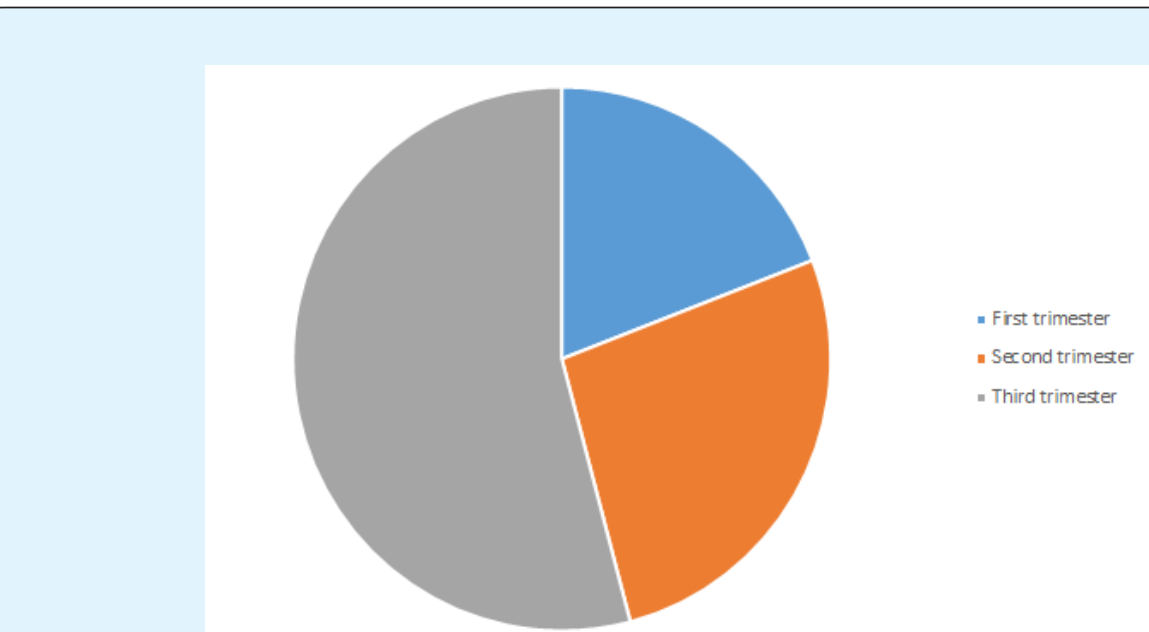

Figure 2: A chart showing the three trimesters and the number of patients per trimester. 


\section{Advances in Pharmacology and Clinical Trials}

Having reviewed the files, the overall distribution of age amongst the patients was between 12 years to 50 years. Most patients were aged 21-30 years 202 (64.3\%) (Figure $1)$.

The trimesters were not evenly distributed amongst the patients, as illustrated in Figure 2.
Findings showed that most women were on their third trimester 170 (54\%). As the patients came for their routinely checkups, they also presented with different medical conditions/complaints, the most common ones being lower abdominal pain 105 (33.4\%) and urinary tract infection 101 (32.2\%), as shown in Figure 3.

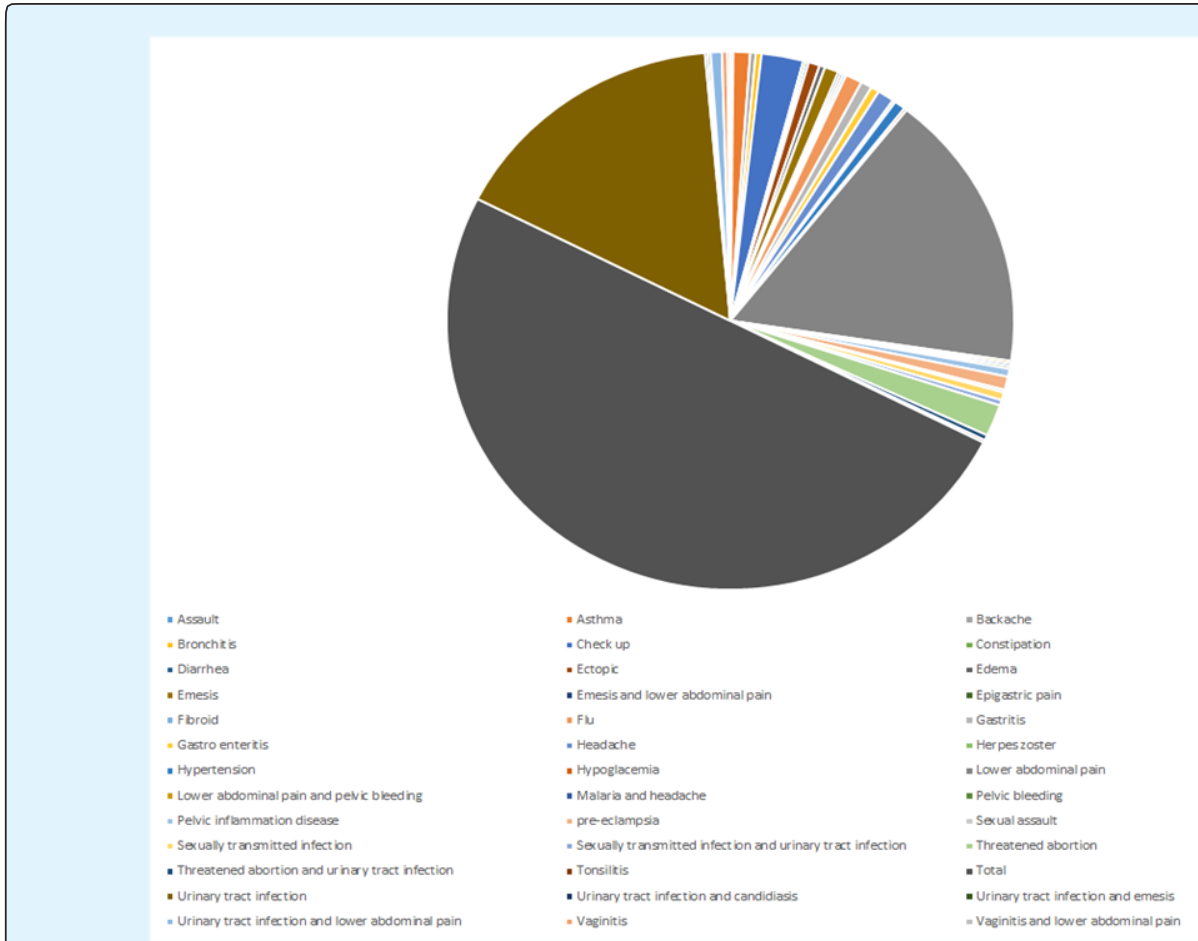

Figure 3: A chart showing medical conditions/complaints the patients presented with.

As illustrated in Figure 4, it was observed that the most prescribed medication for pregnant women were nutritional supplements 682(64.9\%) followed by antimicrobials 188 (17.9\%) and analgesics 127 (12.1\%).

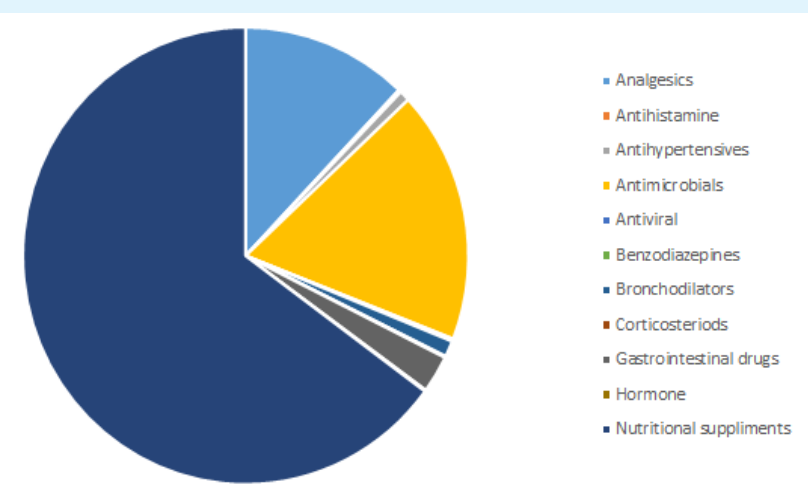

Figure 4: A chart showing classes of drugs prescribed to pregnant women. 


\section{Advances in Pharmacology and Clinical Trials}

Amongst these medications, calcium 242 (23.0\%), folic acid $145(13.8 \%)$ and amoxicillin 105 (10.1\%) were the highly prescribed.
The study showed (Figure 5), that most prescribed drugs were calcium 242 (23.0\%), folic acid 145 (13.8\%), amoxicillin 105 (10.1\%), multivitamins 105 (10.0\%), and paracetamol 87 (8.3\%).

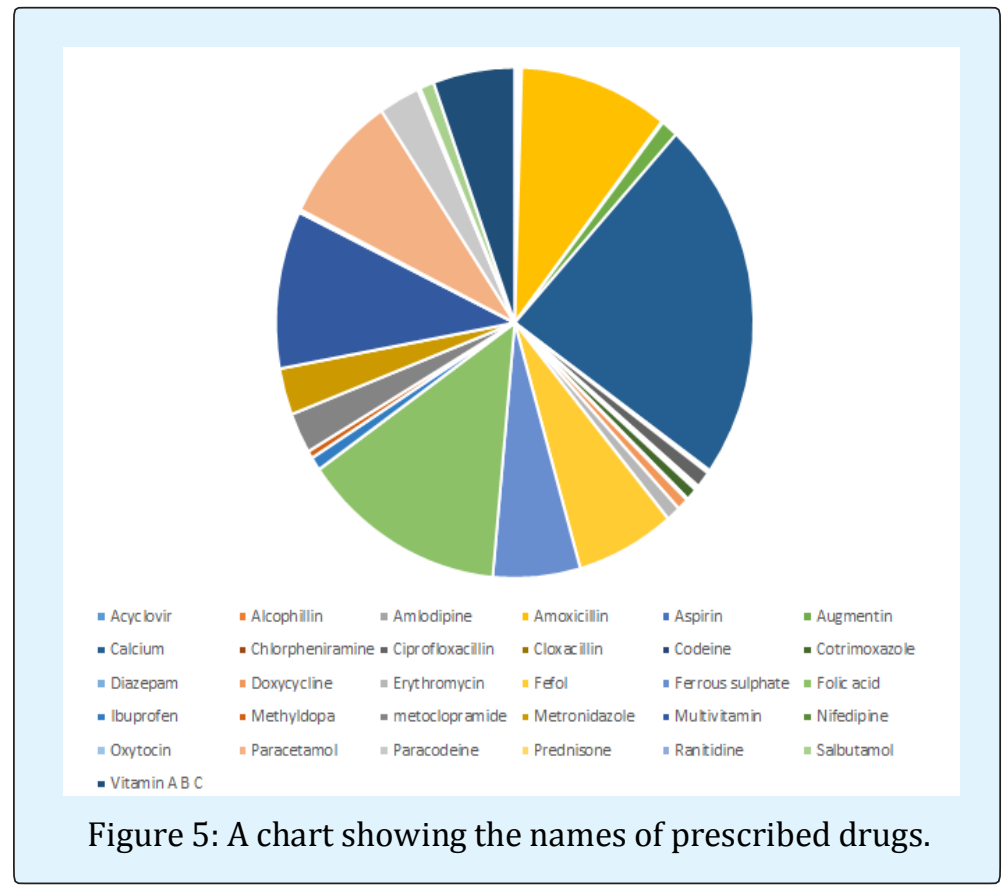

The less prescribed drugs included nifedipine, ranitidine, diazepam, codeine, oxytocin, and aspirin all having value of $1(0.1 \%)$ each amongst others. Doxycycline and cotrimoxazole presented with $8(0.8 \%)$ each.
The medication prescribed to all the 314 pregnant patients were categorized according to the Risk Category classification which consists of five categories in total. Most prescribed drugs fell under category A 686 (64.9\%) and category B 287 (27.3\%), as shown in Figure 6.

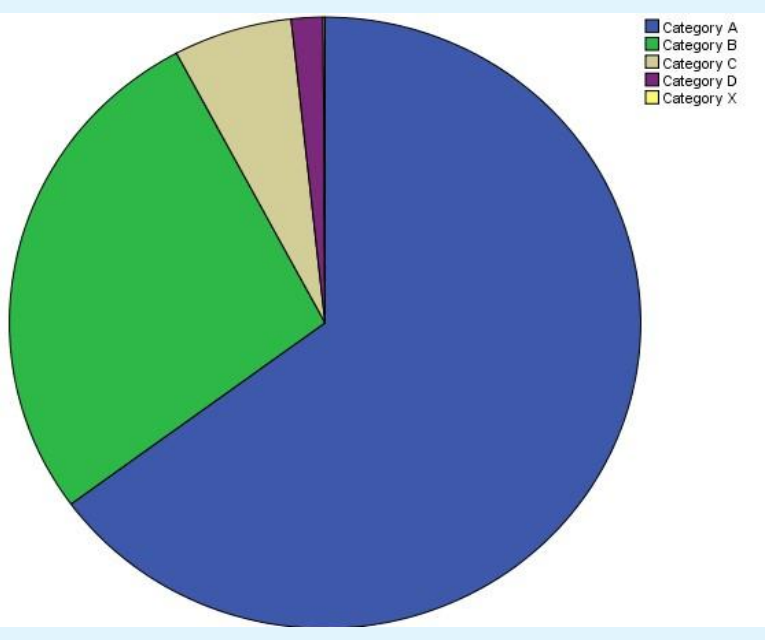

Figure 6: A chart showing drugs prescribed risk category classification. 


\section{Advances in Pharmacology and Clinical Trials}

\section{Discussion}

Out of 314 pregnant women files reviewed, 64.3\% were between 21-30 years, which is the normal reproductive age group. This is in accordance to a study done by Harsh, et al. [14]. Findings of the study showed that a majority of pregnant women were on their third trimester (54\%), yet a study done in Ethiopia contradicted by having most patients on their second trimester $(53.1 \%)$.

This study revealed that most of the pregnant women presented with lower abdominal pain 105 (33.4\%) and urinary tract infection 101(32.2\%). Lower abdominal pain being most prevalent because when the uterus grows and stretches, the ligament that suspend the uterus in the abdomen pull the nearby nerve fibers thus causing pain. As for the urinary tract infection, the foetus grows and sits on the bladder, the increased weight of the foetus blocks the drainage of urine from the bladder thus causing an infection. A study conducted at Sree Siddhartha Medical College in India showed different result [15].

It was observed that the most prescribed drugs were nutritional supplements (64.9\%) having calcium (23\%) and folic acid (13.8\%) as the most prevalent. According to Imdad, et al. [16], calcium reduces neonatal mortality and risk of pre-term birth and folic acid lowers risk of birth defects of the brain and spinal cord (neural tube defects) by up to $70 \%$. A study done in north India by sharma R, Kooper B, and Verma U another in Australia by Etefaand Kahissay [3], and a prospective survey carried out in Southwestern Finland by Heikkila, et al. [17] showed results that contrast with this study.

Amongst the most frequently used antimicrobials, amoxicillin $33.9 \%$ was found to be the most commonly prescribed especially in the treatment of urinary tract infection for pregnant women, it is a category B drug thus is safe for both mother and baby. The study also shows the use of analgesics, third commonly used group of drugs, with paracetamol being highly used because of its demonstration of safety and efficacy at all stages of pregnancy in standard therapeutic doses. Aspirin appears once, being used together with drugs in the treatment of urinary tract infection. The use of aspirin is very low since it has potential risks such as maternal and fetal bleeding through damage of blood vessels in the liver, brain and kidneys [18].

Looking at gastrointestinal drugs used in this study, only ranitidine and metoclopramide were used, metoclopramide being the highest used in this case. Metoclopramide is said to be safe for treating nausea and vomiting during the first trimester of pregnancy, but a study in the USA by Kaunitiz, [19], shows that even when considered being safe, it has potential risk of causing maternal extra pyramidal symptoms. In this study most prescribed drugs fell under category A (64.9\%) and category B (27.3\%). A Similar pattern of category distribution was reported from Netherland, Ethiopia by Zachary, et al. [20] and another study by Joshi, et al. [21]. A study conducted at Sree Siddhartha Medical College in India by Kumarjit, et al. [15], differed from this study by not having category X drugs. Another contrasting study by Tisonova, et al. [22], reported that a vast majority of prescribed drugs during pregnancy, belonged to category C.

Exposure to diazepam, a category D drug, in the first trimester may result in congenital abnormalities in the fetus while third trimester exposure may result in neonatal withdrawal syndrome (Headley, et al.) [18]. In the present study analysis of the drugs taken by pregnant women showed that benzodiazepine anxiolytic diazepam was prescribed to only one pregnant woman. In contrast, a study done in Ethiopia by Mohammed, et al. [5] showed diazepam as the second of twenty most frequently prescribed drugs in pregnancy, used by 303 pregnant women. Prescription analysis study conducted in Pakistan by Das, et al. [23] revealed that less than $1 \%$ of the pregnant women were prescribed teratogenic drugs.

\section{Conclusion}

The overall prescribing and usage pattern in this study was found to be rational, since majority of drugs prescribed were from category $\mathrm{A}$ and $\mathrm{B}$, the safest drugs. Category $\mathrm{C}$ comes third amongst the most used since it possesses potential risks to the foetus and the mother. Category D was the least used since they are used when benefit outweighs risk and in life threatening situations. Category X (oxytocin) drug prescribed was used for the right indication (ectopic pregnancy) in this study.

\section{Recommendations}

It is recommended that prescribers should try to prescribe less category $\mathrm{C}$ and $\mathrm{D}$ drugs as possible with category X not prescribed at all. Adoption and use of US FDA risk category is recommended in Swaziland. There is a need that the same study be conducted in all the regions of Swaziland so that a conclusion can be drawn on if the prescription and use of medication during pregnancy holds the upper hand in causing birth defects in 


\section{Advances in Pharmacology and Clinical Trials}

Swaziland, if not, then the focus can be shifted to other aspects which may possess such effects.

\section{References}

1. Wondesen A, Dabessa G, Belayeneh K G (2016) Drug Use Pattern among Pregnant Women in Adama Hospital Medical College, South East Shewa, Ethiopia. International Journal of Pharma Sciences 6(2): 1426-1435.

2. Uchenna IE, Adego EE, Azuka CO, Ehijie FE (2007) Assessment of prescription Profile of Pregnant Women Visiting Antenatal Clinics. Pharmacy Practice 5(3): 135-139.

3. Etefa K, Kahissay M (2015) Assessment of Drug Prescribing Pattern among Pregnant Women Attending Antenatal Care in Health Centres found in AradaSubcity, Addis Ababa, Ethiopia. Journal of Pharmaceutical Sci 5(4): 347-362.

4. (2016) Ministry of Health Swaziland, Annual Performance Reports. Dissemination Meeting, Monitoring and Evaluation.

5. Mohamme A, Jemal H, Abdulhalik W, Hisham S (2013) Medications use among pregnant women in Ethiopia: A cross sectional study. Journal of Applied Pharmaceutical Science 3(4): 116-123.

6. Dlamini GN, Tiisekwa B (2009) Birth Weight, Stillbirth and Maternal Complications in Swaziland as Related to Parity and Mothers Age. Paper presented at the 7th BOLESWA Symposium on Educational Research for Quality of Life; 2009. The Department of Economics, University of Swaziland Luyengo Campus, M205 Swaziland.

7. WHO (2012) Global estimates on prevalence of hearing loss. Mortality and Burden of Diseases and Prevention of Blindness and Deafness.

8. Ministry of Health (2015) Swaziland National Child Health Report, Expanded Program on Immunization (EPI), Management of Childhood Illness (IMNCI) Program, Nutrition Program.

9. (2015) Ministry of Health Swaziland. Regional Health Performance Report, Manzini Region, Strategic Information Department.

10. (2014) UNICEF Levels and Trends in Child Mortality, Report, Geneva: UNICEF, World Health
Organization, The World Bank, the United Nations Population Division.

11. Wen SW, Yang T, Krewsi D, Yang Q, Nimrod C, et al. (2008) Patterns of pregnancy exposure to prescription FDA C, D and X drugs in a Canadian population. Journal of Perinatology 28 (5): 324-329.

12. Akinsola HA (2005) Research methods in medical \& nursing practice; College Press, Ibadan.

13. Sachdeva P, Patel B (2009) Drug Use in Pregnancy; a Point of Ponder. Indian J Pharm Sci 71(1): 1-7

14. (2012) March of Dimes, PMNCH, Save the Children, WHO. Born Too Soon: The Global Action Report on Preterm Birth. Eds CP Howson. MV Kinney, JE Lawn. World Health Organization. Geneva.

15. Kumarjit S, Manjunath GN, Dhananjaya BS, Lohit K (2015) Prescription pattern of drugs during pregnancy in atertiary care centre: A retrospective study. Journal of International Medicine and Dentistry 2(1): 30-35.

16. Stephansson O, Granath F, Svensson T, Haglund B, Ekbom A (2011) Drug use during pregnancy in Sweden-assessed by the Prescribed Drug Register and the Medical Birth Register. Clinical Epidemiology 3: 43-50.

17. Heikkila AM, Erkkola RU, Nummi SE (2007) Use of medication during pregnancy. A prospective Cohort study on use and policy of prescribing. Ann Chir Gynecol Suppl.

18. Headley J, Northstone K, Simmons H, Golding J (2009) Medication use during pregnancy data from the Avon Longitudinal Study of Parents and Children. Eur Jornal Clin Pharmacol 60: 355-361.

19. (2011) March of Dimes Healthy Babies are Worth the Wait: Preventing Preterm Births through Community-based Interventions: An Implementation Manual. March of Dimes.

20. Zachary B, Moore Z, Govindasamy P (2013) Trends in Demographic and Reproductive indicators in Ethiopia: 2011 demographic and health survey $1^{\text {st }}$ (Edn.), Calverton, Maryland USA: ICF International Inc. 


\section{Advances in Pharmacology and Clinical Trials}

21. United Nations Detention Facility (2015) Millennium Development Goals, Goal 5 Improve Maternal Health Republic of South Africa 4-6

22. Tisonova J, Magulova L, Goboova M (2006) Consultation activity of two Slovak Centres for pharmacotherapy during pregnancy and lactation. Cas Lek Lesk 145: 154-159.
23. Rohra DK, Das N, Azam SI, Solangi NA (2008) Drug prescribing patterns during pregnancy in the tertiary care hospitals of Pakistan a cross sectional study 8: 24 .

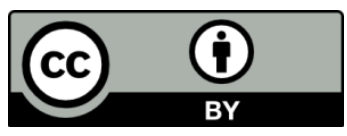

